\title{
Chapter 16 Experimental Validation of Modal Parameters in Rotating Machinery
}

\author{
Bram Vervisch, Kurt Stockman, and Mia Loccufier
}

\begin{abstract}
In this paper, instability of rotating machinery systems due to rotating damping is investigated through experimental modal analysis. Generally, advanced methods are needed for these kind of systems because of the asymmetry of the matrices. However, it is shown by means of cyclic energy dissipation that the rotating damping can be handled as damping which changes due to the rotating speed. The use of damping matrix estimation techniques is discussed and a method is proposed to estimate and model the influence of the rotating damping matrix. A dedicated experimental setup, with negligible gyroscopic effect, is presented for validation purposes. It is shown experimentally that the estimation of the rotating damping matrix is able to predict the decay rate of the first forward mode.
\end{abstract}

Keywords Rotating machinery $•$ Rotating damping - Experimental modal analysis - Damping matrix estimation - Decay rate

\subsection{Introduction}

High speed applications are trending in industry. These applications result in smaller component dimensions and lead to increased production. As a consequence, rotating speeds above the first resonant frequency are more often encountered. A major drawback of this supercritical rotation is instability. At a certain speed, the stability threshold is reached. This stability threshold speed is defined by the ratio between the damping in the nonrotating parts and the damping in the rotating parts [1]. The latter causes, together with the gyroscopic effect, skew-symmetric system matrices and speed dependency. Because of this asymmetry, classical modal analysis techniques are insufficient to describe these systems. Theoretically, decoupling can still be performed by solving the left and right eigenvalue problem [2]. In practice this implies that left and right eigenvectors have to be estimated from measurements. This means that both a row and a column frequency response function matrix have to be measured [3]. In practical applications, generally, not all measurement points can be accessed so an alternative has to be found.

The stability threshold speed of rotating systems is highly dependent on the damping, and damping is very difficult to model. Moreover, the calculation of the stability threshold speed depends on the chosen damping model [4]. Therefore, experimental validation is important. Ideally, an accurate model and some well chosen measurements in combination with a model updating technique can provide a new model that predicts the stability threshold speed. However, model updating proves to be very sufficient for mass and stiffness, but when damping is involved the complexity increases.

In this paper an experimental setup is presented, dedicated to analyze the stability problem and the influence of the rotating damping on the stability threshold speed. A linear speed dependent model is derived to perform the stability analysis. Experimental modal analysis is performed at 0 and 3,000 rpm. It is shown that by treating the system as nonrotating at both speeds still leads to an estimation of the rotating damping effect. As such, insight is gained in the physical phenomenon of rotating damping and its influence on the real part of the poles or the decay rate. In Sect. 16.2, experimental modal analysis of

B. Vervisch $(\triangle)$

Department of Industrial Systems and Product Design, Ghent University, Graaf Karel de Goedelaan 5, 8500 Kortrijk, Belgium

Department of Electrical Engineering, Systems and Automation, Ghent University, Technologiepark 914, 9000 Ghent, Belgium

e-mail: bramf.vervisch@ugent.be

K. Stockman

Department of Industrial Systems and Product Design, Ghent University, Graaf Karel de Goedelaan 5, 8500 Kortrijk, Belgium

M. Loccufier

Department of Electrical Engineering, Systems and Automation, Ghent University, Technologiepark 914, 9000 Ghent, Belgium 
rotating systems is discussed and it is shown that because of the cyclic energy dissipation, the rotating damping can be treated as regular damping that is only speed dependent. In Sect. 16.3, the estimation of the damping matrix is discussed. In Sect. 16.4 the rotating damping setup is presented and a method to extract the rotating damping effect is proposed. Section 16.5 provides an experimental validation.

\subsection{Experimental Modal Analysis of Rotating Systems}

\subsubsection{The Linear Speed Dependent Model}

The model used in this paper includes speed dependency by means of rotating damping. The gyroscopic effect is neglected for a rotor symmetric in $\mathrm{x}$ and $\mathrm{y}$ direction the equations of motion can be written as

$$
\left[\begin{array}{cc}
\mathbf{M} & \mathbf{0} \\
\mathbf{0} & \mathbf{M}
\end{array}\right]\left\{\begin{array}{l}
\ddot{\mathbf{x}} \\
\ddot{\mathbf{y}}
\end{array}\right\}+\left[\begin{array}{cc}
\mathbf{C}_{n}+\mathbf{C}_{r} & \mathbf{0} \\
\mathbf{0} & \mathbf{C}_{n}+\mathbf{C}_{r}
\end{array}\right]\left\{\begin{array}{l}
\dot{\mathbf{x}} \\
\dot{\mathbf{y}}
\end{array}\right\}+\left(\left[\begin{array}{cc}
\mathbf{K} & \mathbf{0} \\
\mathbf{0} & \mathbf{K}
\end{array}\right]+\Omega\left[\begin{array}{cc}
\mathbf{0} & \mathbf{C}_{r} \\
-\mathbf{C}_{r} & \mathbf{0}
\end{array}\right]\right)\left\{\begin{array}{l}
\mathbf{x} \\
\mathbf{y}
\end{array}\right\}=\left\{\begin{array}{l}
\mathbf{F}_{x} \\
\mathbf{F}_{y}
\end{array}\right\}
$$

where $\mathbf{M}$ and $\mathbf{K}$ are the regular mass and stiffness matrices. $\mathbf{C}_{n}$ is the nonrotating damping or the damping that only influences the nonrotating frame. The matrix $\mathbf{C}_{r}$ includes the rotating damping or the damping that influences the rotating frame. $\mathbf{C}_{r}$ depends on the rotating speed $\Omega$. The vector $\left\{\begin{array}{ll}\mathbf{x} & \mathbf{y}\end{array}\right\}^{T}$ represents the generalized coordinates in $\mathrm{x}$ - and y direction. As can be seen, the model not only deviates from a classical mass spring damper system by the speed dependency but both gyroscopic effect and rotating damping provide a coupling between $\mathrm{x}$ and $\mathrm{y}$. Moreover, this coupling is skew-symmetric, which yields a difficulty in the use of classical modal analysis where symmetric matrices are assumed.

\subsubsection{Cyclic Energy Dissipation and Stability}

The appearance of skew-symmetric matrices have a particular effect on the energy dissipation and stability of the system as explained by Adams in [5] and [6]. Consider the equations of motion for a system with only two degrees of freedom

$$
\left[\begin{array}{cc}
m & 0 \\
0 & m
\end{array}\right]\left\{\begin{array}{l}
\ddot{x} \\
\ddot{y}
\end{array}\right\}+\left(\left[\begin{array}{cc}
c_{n}+c_{r} & 0 \\
0 & c_{n}+c_{r}
\end{array}\right]+\Omega\left[\begin{array}{cc}
0 & g \\
-g & 0
\end{array}\right]\right)\left\{\begin{array}{l}
\dot{x} \\
\dot{y}
\end{array}\right\}+\left(\left[\begin{array}{cc}
k & 0 \\
0 & k
\end{array}\right]+\Omega\left[\begin{array}{cc}
0 & c_{r} \\
-c_{r} & 0
\end{array}\right]\right)\left\{\begin{array}{l}
x \\
y
\end{array}\right\}=\left\{\begin{array}{l}
F_{x} \\
F_{y}
\end{array}\right\}
$$

The work done by the displacement term or apparent stiffness term of the rotating damping can be expressed as

$$
\begin{array}{r}
d w=-\Omega\left[\begin{array}{rr}
0 & c_{r} \\
-c_{r} & 0
\end{array}\right]\left\{\begin{array}{l}
x \\
y
\end{array}\right\}\{d x d y\}=\Omega\left(-c_{r} y d x+c_{r} x d y\right) \equiv f_{x} d x+f_{y} d y \\
\therefore \frac{\partial f_{x}}{\partial y}=-\Omega c_{r} \quad \text { and } \quad \frac{\partial f_{x}}{\partial x}=\Omega c_{r}
\end{array}
$$

Because $\partial f_{x} / \partial y \neq \partial f_{y} / \partial x, \mathrm{dw}$ is no exact differential, leading to a nonconservative force field. This means that the rotating damping appearing as a stiffness term actually dissipates energy. Together with the regular damping, the sum of the nonconservative forces of the rotating system can be written as

$$
\left\{\begin{array}{l}
P_{x} \\
P_{y}
\end{array}\right\}=-\left[\begin{array}{cc}
c_{n}+c_{r} & 0 \\
0 & c_{n}+c_{r}
\end{array}\right]\left\{\begin{array}{l}
\dot{x} \\
\dot{y}
\end{array}\right\}-\Omega\left[\begin{array}{cc}
0 & c_{r} \\
-c_{r} 0
\end{array}\right]\left\{\begin{array}{l}
x \\
y
\end{array}\right\}
$$

Suppose that the rotor is operated at a certain speed $\Omega$ and whirling at $\omega$. The whirling can be expressed as a harmonic motion in $\mathrm{x}$ and $\mathrm{y}$ direction, $x=X \sin \left(\omega t+\phi_{x}\right)$ and $y=Y \sin \left(\omega t+\phi_{y}\right)$. The energy per cycle that is exchanged due to these forces can be expressed as

$$
E_{c y c}=\oint P_{x} d x+P_{y} d y=\int_{0}^{2 \pi / \omega}\left(P_{x} \dot{x} d t+P_{y} \dot{y} d t\right)
$$




$$
E_{c y c}=-\pi[\underbrace{\omega\left(c_{n}+c_{r}\right)\left(X^{2}+Y^{2}\right)}_{\text {regular damping effect }}-\underbrace{2 \Omega X Y \sin \left(\phi_{x}-\phi_{y}\right)}_{\text {speed dependent effect }}]
$$

This is an important expression because it shows that the appearance of rotating damping can lead to a positive energy per cycle or instability. The first term is a regular damping effect and causes a cyclic decrease of the energy. The second term is not only speed dependent, but also depends on the phase difference between $\phi_{x}$ and $\phi_{y}$. Whenever the whirling $\omega$ occurs in the same direction as the rotation $\Omega$, which is called a forward mode, $\sin \left(\phi_{x}-\phi_{y}\right)>0$. As $\Omega$ increases the influence of the second term in (16.6) increases. When the energy per cycle becomes positive, instability occurs. For backward modes, $\sin \left(\phi_{x}-\phi_{y}\right)<0$, and the effect of the second term in (16.6) is always stabilizing.

\subsubsection{Modeling of Rotating Damping}

Although the gyroscopic effect plays an important role in rotating machinery, it is omitted in this paper since the focus lies on the rotating damping. When there is no gyroscopic effect, (16.1) can be written as

$$
\mathbf{M} \ddot{\mathbf{q}}+\left(\mathbf{C}_{n}+\mathbf{C}_{r s}\right) \dot{\mathbf{q}}+\left(\mathbf{K}+\Omega \mathbf{C}_{r s s}\right) \mathbf{q}=\mathbf{F}
$$

where $\left\{\begin{array}{ll}\mathbf{x} & \mathbf{y}\end{array}\right\}^{T}$ is combined in $\mathbf{q}$ and therefore a distinction is made between the symmetric part of the rotating damping $\mathbf{C}_{r s}$ and the skew-symmetric part $\mathbf{C}_{r s s}$. The appearance of the rotating damping as an apparent stiffness term has a relevant physical meaning [7] but leads to experimental difficulties. In order to decouple this system both left and right eigenvalue problem have to be solved. In a theoretical model this is possible, but in practice it implies that left and right eigenvectors have to be estimated from measurements. This is experimentally not always possible, thus this approach is difficult to apply. By slightly deviating from the exact physical model, an experimentally more interesting model is proposed

$$
\mathbf{M} \ddot{\mathbf{q}}+\left(\mathbf{C}+\Omega \mathbf{C}_{r e}\right) \dot{\mathbf{q}}+\mathbf{K q}=\mathbf{F}
$$

The physical relevance of this equation lies in (16.6) and the fact that all forms of energy dissipation are modeled in the damping matrix. The damping at standstill is combined in $\mathbf{C}$ and the difference in damping due to rotation is combined in $\mathbf{C}_{r e}$. If both $\mathbf{C}$ and $\mathbf{C}_{r e}$ are estimated from measurements, the effect of the rotating speed on the damping can be predicted.

\subsection{Estimation of the Damping Matrix}

\subsubsection{Damping Matrix Estimation Methods}

Identification of damping from measurements is an important topic in literature. Especially when it involves the identification of the damping matrix. Damping matrix identification methods are classified in matrix methods and modal methods [8]. Matrix methods are normally based upon the measurement of the whole frequency response function matrix and modal methods use modal parameters. The latter is preferred in this research because only one row of the frequency response function matrix is needed which highly limits the number of measurements. One of the modal methods is developed by Adhikari in a series of papers. Only the viscous damping identification method is used here [9]. The main advantage of this method is that it allows asymmetry of the matrices and that the reconstructed physical matrix has acceptable spatial distribution. It can also deal with errors due to modal truncation and noise. 


\subsubsection{Limitations of the Damping Estimation Method}

\subsubsection{Mode Shape Errors}

Modal methods rely on an exact knowledge of the mode shapes and are extremely sensitive to errors [10-12]. However, several attempts have been made to optimize the damping matrix even with erroneous mode shapes. Pilkey [13] uses an iteration technique combined with Lancasters method to optimize the damping matrix. The main drawback is that the mass matrix has to be known. When symmetry of the damping matrix is a preliminary assumption, Adhikari proposes a symmetry preserving method, which forces experimental data to yield a symmetric matrix [14]. Balmès [15] uses Lancasters reconstruction method to define the orthogonality property between the eigenvectors, known as the properness condition. Subsequently an optimization procedure is used to find the eigenvectors that meet this properness condition.

The scaling of the mode shapes in Adhikari's method is unity modal mass scaling or

$$
\mathbf{X}^{*} \mathbf{M X}=\mathbf{I}
$$

with $\mathbf{X}$ the matrix containing the mode shapes or eigenvectors, $\mathbf{I}$ is the identity matrix and () ${ }^{*}$ denotes the conjugate transpose. So when the mass matrix is known, the mode shapes can be rescaled to unity modal mass yielding a better estimation. With finite elements it is possible to estimate mass matrices quite accurately. In order to improve the mass matrix even more, model updating techniques can be used.

\subsubsection{Incomplete Data and Modal Truncation}

When performing experimental modal analysis, the measured data is always incomplete. Due to the limited bandwidth of both excitation and response, modal truncation occurs. The poles and mode shapes that result from a measurement are limited by this bandwidth. Also, not all degrees of freedom that are assumed in the model can be measured, especially rotational degrees of freedom. Truncated poles and mode shapes can still be used in Adhikari's method but the limited degrees of freedom limit the dimension of the resulting damping matrix.

\subsection{Extracting the Rotating Damping Effect from Measurements}

\subsubsection{The Rotating Damping Setup}

The rotating damping setup is described in [16]. The purpose of the setup is a shaft that has clamped boundary conditions on both sides. The setup is shown schematically in Fig. 16.1. Five measurement locations are chosen equally divided along the shaft. The motion inside the bearings is difficult to measure so this is avoided. These five measurement locations are sufficient to identify the first five poles and mode shapes.

The shaft can be operated at a speed up to $6,160 \mathrm{rpm}$. For safety purposes, the range is kept between 0 and 4,500 rpm. As excitation, an impact hammer is used. Because impact testing requires an experienced operator, an automated impact is designed (Fig. 16.2). The responses are measured with eddy current probes. These probes typically generate a noisy signal and therefore every measurement is an average in frequency domain of ten impacts. The frequency response functions are estimated with an $\mathrm{H} 1$ estimator [17].

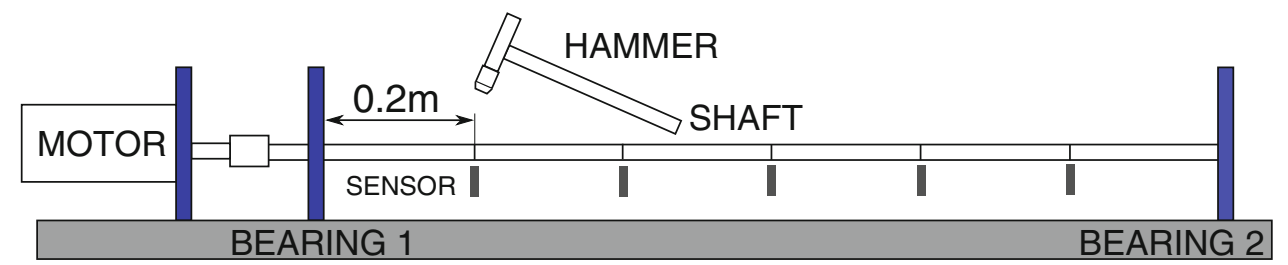

Fig. 16.1 The rotating damping setup is the equivalent of a clamped beam on both sides from which five measurement points are taken 
Fig. 16.2 The impact hammer is automated by means of a stepping motor that is controlling the hit in order to improve the reproducibility
Fig. 16.3 Each element of the finite element model has eight degrees of freedom
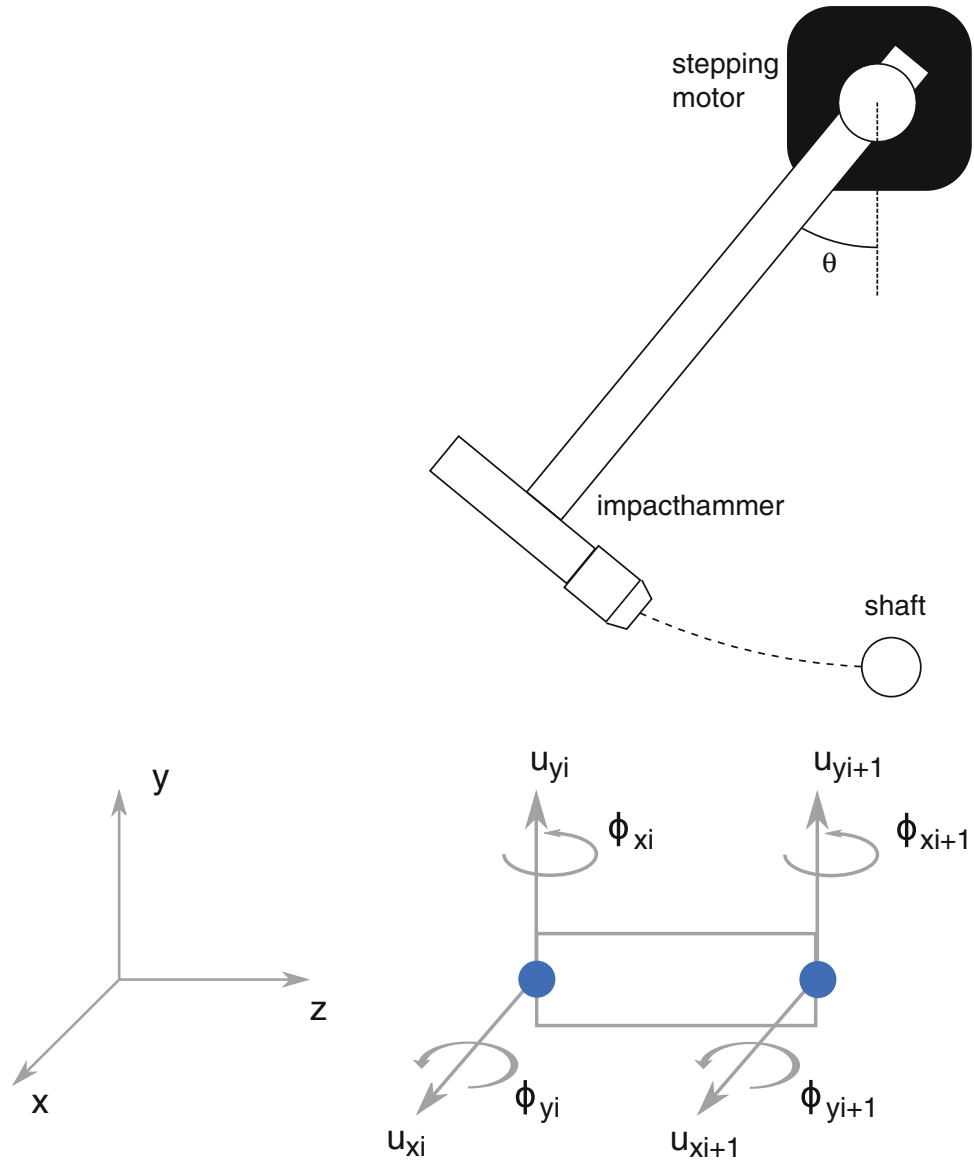

\subsubsection{Nonrotating Shaft}

\subsubsection{Modeling, Updating and Reducing of the Model}

When the shaft is not rotating, there are no speed dependent effects, so a classical mass, spring damper system is assumed. The shaft is modeled with finite elements [18]. Six elements used, with each eight degrees of freedom (Fig. 16.3), so the resulting model has a dimension of $28 \times 28$. Only five frequency response functions are measured. The model updating method applied here [19] is based upon the frequency response functions and can deal with incomplete data by using an iterative procedure. The mass and the stiffness matrix of the model are updated and the dimension remains $28 \times 28$. Consequently, the (purely imaginary) poles and the corresponding (real) mode shapes of this undamped model are calculated. Because there are only five frequency response functions in the measurement, it is only possible to derive a damping matrix with dimension $5 \times 5$. Therefore, the modeled mass and stiffness matrices are reduced to a dimension of $5 \times 5$. This is done by truncating both calculated poles and mode shapes to the first five natural frequencies and by deleting the coordinates in the mode shapes that cannot be measured. By doing this the dimensions of the calculated poles and mode shapes correspond to the measurement. The resulting poles and mode shapes are used to reconstruct a new mass and stiffness matrix by transforming the modal matrices back to physical coordinates.

\subsubsection{Identification of the Damping Matrix}

The previous description results in updated mass and stiffness matrices with a dimension of $5 \times 5$. Next, the measured frequency response functions are used to extract poles and mode shapes with a least squares complex exponential method. The extracted mode shapes are unity modal mass scaled using the reduced mass matrix from the model. The newly scaled mode shapes are used together with the poles to estimate the damping matrix $\mathbf{C}_{0}$. 


\subsubsection{Rotating Shaft}

When the shaft is rotating at a certain speed it behaves like the system in (16.1). However, if a system as (16.8) is assumed, then all the dissipation effects are combined in the damping matrix. This means that the $5 \times 5$ mass and stiffness matrix of the rotating system are not changing due to rotation. The measured frequency response functions at rotating speed $\Omega$ are then used to extract the poles and mode shapes. The resulting mode shapes are erroneous, so they are scaled to unity modal mass using the mass matrix derived at 0 speed. These scaled mode shapes are then used to estimate a damping matrix of the form $\mathbf{C}_{\Omega}=\mathbf{C}+\Omega \mathbf{C}_{r e}$. The rotating damping effect is now estimated by

$$
\mathbf{C}_{r e}=\frac{C_{\Omega}-\mathbf{C}_{0}}{\Omega}
$$

\subsection{Experimental Validation}

In the experiment, the shaft is operated at 0 and 3,000 rpm. At both speeds, the frequency response functions are measured. The identified damping matrices are given in Tables 16.1 and 16.2. The damping matrices are not symmetric and there are negative elements on the diagonal. However, together with the updated mass and stiffness matrix, they can be used to estimate the frequency response functions accurately. The measured and estimated frequency response functions at the driving point are given in Figs. 16.4 and 16.5. Because of the impact measurement, an exponential window has been used to estimate

Table 16.1 The identified damping matrix at $0 \mathrm{rpm}$

\begin{tabular}{c|c|c|c|c}
\hline 14.98719 & -3.7457 & 17.42148 & 5.822773 & 10.20963 \\
\hline-4.36665 & -9.92687 & -15.0152 & -13.9544 & -11.8712 \\
\hline 7.730844 & 6.800062 & 24.19674 & 11.88099 & 16.13112 \\
\hline-1.65739 & -17.3205 & -6.53153 & -20.9999 & -13.4505 \\
\hline 16.69047 & -14.5339 & 27.78173 & -2.4798 & 13.98526 \\
\hline
\end{tabular}

Table 16.2 The identified damping matrix at $3,000 \mathrm{rpm}$
Fig. 16.4 The frequency response function taken at the driving point at $0 \mathrm{rpm}$ can be reconstructed by using the identified damping matrix

\begin{tabular}{c|c|c|c|c}
\hline-13.2302 & -36.2871 & -25.6383 & -5.76573 & -4.88674 \\
\hline 24.98378 & 26.9026 & 26.24019 & 8.278528 & 5.051465 \\
\hline-25.6916 & -7.65284 & -34.3064 & -12.0996 & -4.71555 \\
\hline-9.68825 & 33.90984 & 9.023721 & 16.4902 & -1.24859 \\
\hline-4.99275 & -5.65304 & -8.7208 & -5.00681 & 4.067461 \\
\hline
\end{tabular}

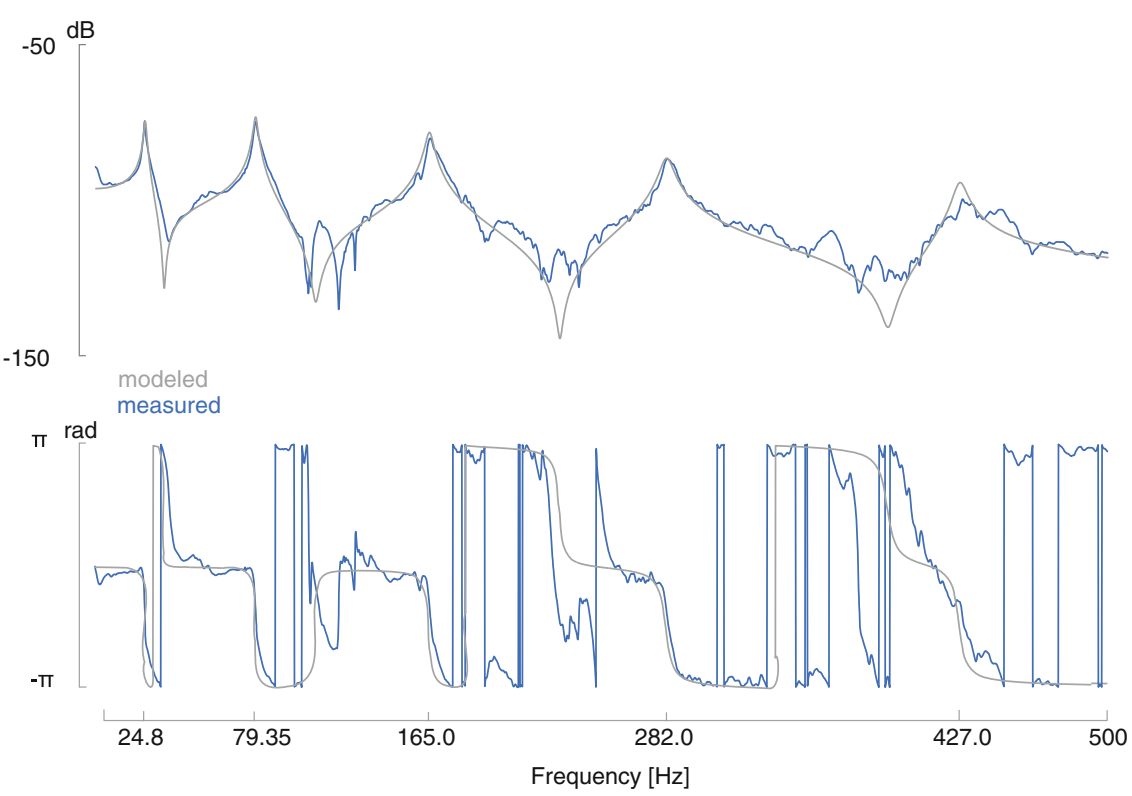


Fig. 16.5 The frequency response function taken at the driving point at $3,000 \mathrm{rpm}$ can be reconstructed by using the identified damping matrix

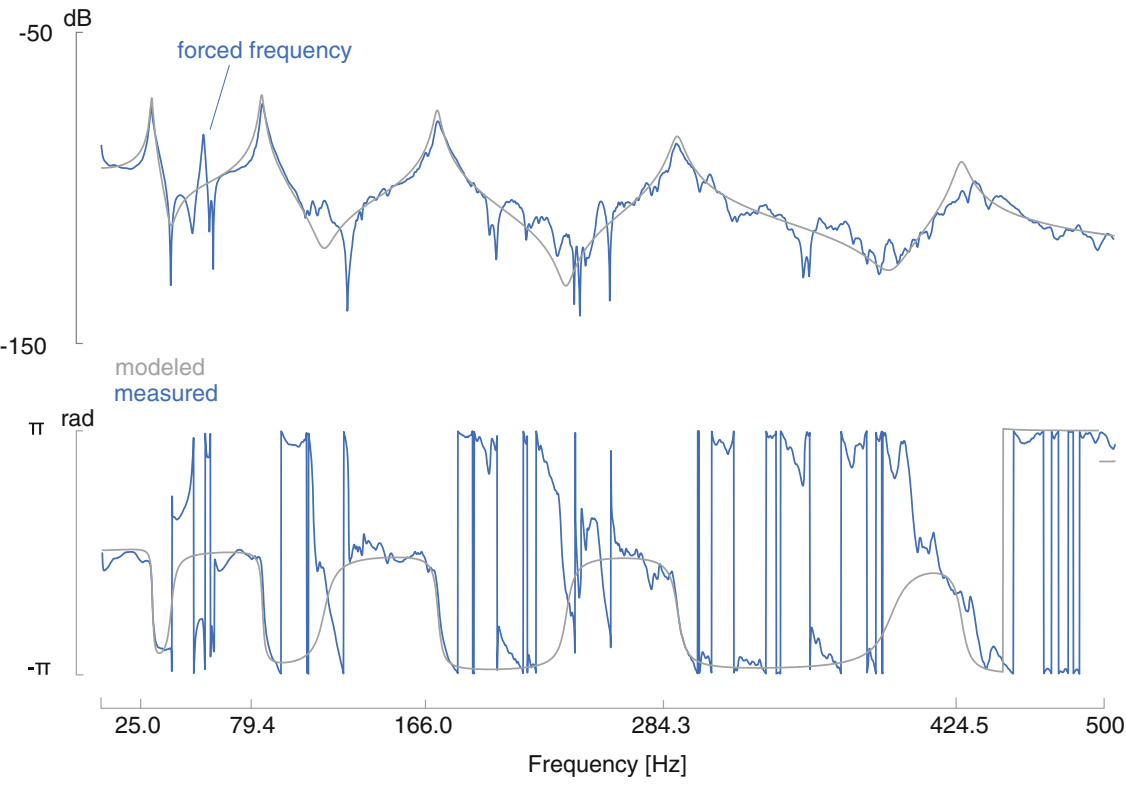

the frequency response functions. After compensation of this window effect [17], the estimated damping is slightly lower. At 3,000 rpm, there is an unavoidable peak that is caused by the residual unbalance of the shaft.

The estimated mass, damping and stiffness matrices are used to predict the decay rate plot of the first forward mode (Fig. 16.6). In order to validate the prediction the system is operated at several speeds going from 0 to $4,500 \mathrm{rpm}$ with a resolution of $500 \mathrm{rpm}$. The measurement at $1,500 \mathrm{rpm}$ is not performed because it was too close to the first natural frequency leading to unwanted high amplitudes. At each speed, the real part of the poles is estimated and compared to the prediction. As can be seen, the prediction fits remarkably well. It should be noted that whereas the model predicts that the system will become instable. This prediction indicates that the first forward mode will not cause instability. At about 3,000 rpm, there seems to be some sort of stabilizing effect.

\subsection{Conclusion}

In this paper the phenomenon of rotating damping is investigated through experiments. Instead of approaching the system as skew-symmetric a different approach is proposed. A skew-symmetric model is experimentally not convenient. When the energy loss per cycle is observed, the rotating damping that appears in the displacement term or apparent stiffness term, actually behaves as regular damping term that is speed dependent. Therefore, a rotating system can be observed as a classical mass spring damper system that has just a speed dependency of the damping matrix.

A rotating damping setup is used to validate the proposed method. In combination with a finite element model and a model updating technique, the damping matrix is estimated both at 0 and 3,000 rpm. The difference between the two damping matrices is a measure for the rotating damping effect. By using the estimated damping matrices together with the updated mass and stiffness matrices, the decay rate plot of the first forward mode is predicted. The prediction is validated with single measurements at different speeds. It is important to notice that a linear speed dependent model with viscous damping predicts a certain stability threshold speed. The prediction in this paper however shows that there appears to be a stabilizing effect. The same effect is validated in the single measurements. In this research, both nonrotating and rotating damping are very low because of the configuration of the setup. In future, the influence of extra damping can be investigated together with the inclusion of the gyroscopic effect. 


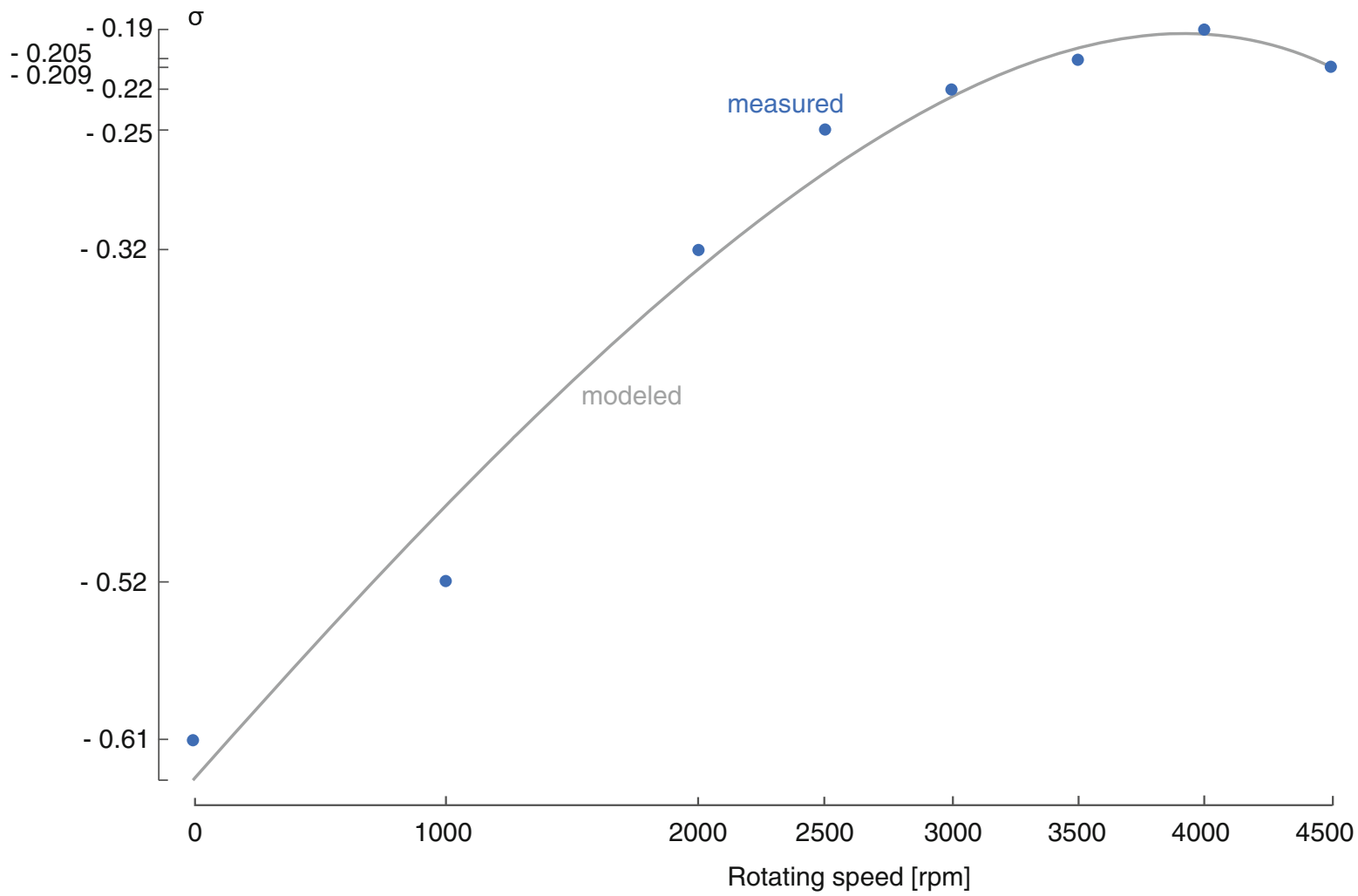

Fig. 16.6 The decay rate plot is constructed with the identified damping matrices at 0 and 3,000 rpm. The results are validated by individual measurements at different speeds

\section{References}

1. Vervisch B, Stockman K, Loccufier M (2014) Estimation of the damping matrix in rotating machinery for the calculation of the stability threshold speed. Int J Struct Stab Dyn 14:1450012

2. Tisseur F, Meerbergen K (2006) The quadratic eigenvalue problem. Soc Ind Appl Math 43(2):235-286

3. Bucher I, Ewins DJ (2001) Modal analysis and testing of rotating structures. Philos Trans R Soc A Math Phys Eng Sci 359(1778):61-96

4. Vervisch B, Stockman K, Loccufier M (2012) Sensitivity of the stability threshold in linearized rotordynamics. In: ISMA conference, Leuven

5. Adams ML (2009) Rotating machinery vibration: from analysis to troubleshooting. CRC Press/Taylor \& Francis, Boca Raton

6. Adams ML, Padovan J (1981) Insights into linearized rotor dynamics. J Sound Vib 76(1):129-142

7. Genta G (2005) Dynamics of rotating systems, vol 1. Springer, New York

8. Srikantha Phani A, Woodhouse J (2007) Viscous damping identification in linear vibration. J Sound Vib 303(3-5):475-500

9. Adhikari S, Woodhouse J (2001) Identification of damping: part 1, viscous damping. J Sound Vib 243(1):43-61

10. Adhikari S, Woodhouse J (2002) Identification of damping: part 4, error analysis. J Sound Vib 251(3):491-504

11. Srikantha Phani A, Woodhouse J (2009) Experimental identification of viscous damping in linear vibration. J Sound Vib 319(3-5):832-849

12. Foltête E, Gladwell GML, Lallement G (2001) On the Reconstruction of a damped vibrating system from two complex spectra, part 2: experiment. J Sound Vib 240(2):219-240

13. Pilkey DF, Inman DJ (1997) An iterative approach to viscous damping matrix identification. In: IMAC XV proceedings, pp $1152-1157$

14. Adhikari S, Woodhouse J (2002) Identification of damping: part 3, symmetry-preserving methods. J Sound Vib 251(3):477-490

15. Balmes E (1997) New results on the identification of normal modes from experimental complex modes. Mech Syst Signal Process 11(2):229_ 243

16. Vervisch B, Derammelaere S, Stockman K, Loccufier M (2014) Frequency response functions and modal parameters of a rotating system exhibiting rotating damping. In: ISMA conference 2014

17. Brandt A (2011) Noise and vibration analysis: signal analysis and experimental procedures. Wiley, Chichester

18. Forrai L (2000) A finite element model for stability analysis of symmetrical rotor systems with internal damping. JCAM 1(1):37-47

19. Lin RM, Ewins DJ (1994) Analytical model improvement using frequency response functions. Mech Syst Signal Process 